PLEASE NOTE:

This is the author's version of the manuscript accepted for publication in Psychology \& Health. Changes resulting from the publishing process, namely editing, corrections, final formatting for printed or online publication, and other modifications resulting from quality control procedures, may have been subsequently added.

The published version can be found in: Brandão, T., Schulz, M. S., \& Matos, P. M. (2014). Psychological intervention with couples coping with breast cancer: A systematic review. Psychology \& Health, 29(5), 491-516. doi: 10.1080/08870446.2013.859257 


\section{Psychological intervention with couples coping with breast cancer: A systematic review}

Tânia Brandão $^{a}$, Marc S. Schulz ${ }^{b} \&$ Paula Mena Matos ${ }^{a}$

a Faculty of Psychology and Education, University of Porto; Center for Psychology at University of Porto; Porto, Portugal.

${ }^{\mathrm{b}}$ Department of Psychology, Bryn Mawr College, Pennsylvania, USA.

*Corresponding author contact information: Dr. Paula Mena Matos, Rua Alfredo Allen 4200-135 Porto Portugal; Email: pmmatos@fpce.up.pt

Funding for this study was provided by a PhD Scholarship from the Portuguese Foundation for Science and Technology (FCT) granted to the first author (SFRH/BD/84436/2012).

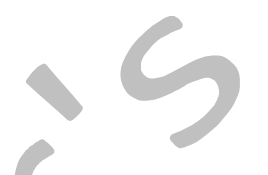

\footnotetext{
Abstract

Objective: Information about psychological intervention with couples coping with breast cancer is not well-disseminated. This can be explained, at least in part, by the absence of knowledge about the efficacy of this kind of intervention. The aim of the present systematic review is to identify and describe psychological interventions for couples coping with breast cancer and evaluate their efficacy.

Design: Studies identified by a searching multiple literature databases related to health and psychology between 1975 and 2013. Rigorous inclusion and exclusion criteria were utilized.

Results: Of 129 abstracts, 13 were extracted for further analysis and a final ten studies were deemed eligible for inclusion. Data were extracted from each study regarding study sample characteristics, design, results and methodological limitations. The results obtained were mixed in regard to efficacy, although the overwhelming majority of studies (eight studies) found benefits for both women and their partners in some dimensions, such
} 
as quality of life, psychological distress, relationship functioning and physical symptoms associated with cancer.

Conclusion: Psychological interventions for couples coping with breast cancer appear to be effective for both women and their partners. However, further studies are needed to evaluate the efficacy of couple-based interventions and, to identify for whom and how they are more effective.

Keywords: breast cancer; systematic review; couple intervention

\section{Introduction}

Breast cancer is the most common type of cancer for women in Europe and in the World. In Europe, in 2012 an estimated 464.000 new cases were diagnosed and 131.000 women died from this disease (Ferlay et al., 2013). The diagnosis and associated treatments can be a traumatic experience for women. Breast cancer can challenge patients' fundamental beliefs regarding personal vulnerability, safety, and self-worth. Patients often experience anxiety, depression, uncertainty, decreased quality of life, concerns about body image, and specific difficulties associated with physical symptoms (Anagnostopoulos, Slater, \& Fitzsimmons, 2010; Badger, Segrin, Dorros, Meek, \& Lopez, 2007; Montazeri et al., 2008; Moreira \& Canavarro, 2010; Silva, Bettencourt, Moreira, \& Canavarro, 2011). The effects of breast cancer, however, extend beyond the individual patient since breast cancer initiates challenges and distressing consequences not only for patients but also for their loved ones, particularly life partners. In fact, compared to men from the general population, husbands of women with breast cancer report lower quality of life and higher levels of depression and anxiety and feelings of helplessness, exhaustion and fear (Badger et al., 2007; Lethborg, Kissane, \& Burns, 2003; Moreira \& Canavarro, 2013; Wagner, Bigatti, \& Storniolo, 2006).

Research shows that women with breast cancer identify their partners as their main source of support and that this support plays an important role in women's psychological well-being and adjustment to the disease as well as in their physical health (e.g., immune function) (Baider, Ever-Hadani, Goldzweig, Wygoda, \& Peretz, 2003; Baucom et al., 2012; Manne et al., 2005; Stiell, Naaman, \& Lee, 2007; Weihs, Enright, \& Simmens, 2008). However, partners are not always able to respond in a helpful and supportive way because of their own psychological distress, problems in the couple relationship, or complicated motivations about caregiving (e.g., acting to avoid feeling guilt, following social expectations about a proper role, or to find a sense of purpose and 
fulfillment in caregiving task). In fact, motives for caregiving influence not only the quantity and quality of the support provided (more or less responsive or effective) but also the well-being of the support provider (Feeney \& Collins, 2003; Kim, Carver, Deci, \& Kasser, 2008).

The dyadic challenges created by the presence of a breast cancer diagnosis and subsequent treatment can also lead to relational distress and difficulties in couples' communication, sexual functioning and intimacy (Manne et al., 2006; Sbitti et al., 2011; Stiell et al., 2007; Ussher, Perz, \& Gilbert, 2012). For these reasons, it is important to develop and implement psychological interventions for couples coping with breast cancer, incorporating partners actively as participants in these interventions.

In the last decade some work has been done in this area. Some psychological programs have been developed for couples coping with breast cancer (e.g., Baucom et al., 2009; Manne et al., 2005; Scott, Halford, \& Ward, 2004). There is, however, little information about the effectiveness of psychological interventions for couples coping with breast cancer. Previous systematic reviews and meta-analyses have tended to lump together couples interventions for a broad mix of cancers (e.g., breast, prostrate, lung) or to include interventions for other types of chronic diseases (e.g., cardiovascular disease or hypertension, osteoarthritis, HIV, diabetes) (Brad \& Krebs, 2012; Martire et al., 2010; Regan et al., 2012). Some systematic reviews have focused on other types of dyads coping with cancer, such as parent-child dyads (Northouse et al., 2010).

Past systematic reviews and meta-analyses indicate that psychological intervention for couples coping with a variety of cancer diagnoses or other serious chronic diseases are generally effective for both patients and their partners. Badr and Krebs (2012) found that psychological interventions for couples confronting different types of cancer (mainly breast and prostate cancer) are effective in improving the quality of life of patients (Hedges $g=0.25$ for psychological variables, $g=0.31$ in physical variables and $\mathrm{g}=0.28$ in relational variables) and their partners $(\mathrm{g}=0.21$ in psychological variables and $g=0.24$ in relational variables). Martire et al. (2010) found that psychological interventions for couples coping with a variety of chronic disease (e.g., cancer, chronic pain or HIV) have positive benefits for patients, including reducing depressive symptoms (Cohen's $d=0.18)$, improving marital functioning $(d=0.17)$ and reducing pain $(d=0.19)$. However, none of the systematic reviews and meta-analyses have focused only on couples coping with breast cancer. 
Thus, the main objective of this systematic review was to analyze the content, the format, as well as the efficacy of psychological interventions for couples coping with breast cancer, in order to help inform future intervention efforts in this area. In this review, inclusion criteria include only studies that examine psychological interventions with adult couples coping with breast cancer. This systematic review was informed by the Cochrane Collaboration guidelines (Higgins \& Green, 2011).

\section{Method}

Eligibility criteria

Criteria for considering studies for this review were: (1) randomized studies that analyse the efficacy of psychological interventions for women coping with breast cancer; (2) the intervention studied must have a couple component that includes the partner (i.e., the spouse or intimate partner must be included in the intervention); (3) health, psychological and/or relationship outcomes must be evaluated. Exclusion criteria were: (1) studies without a comparison condition (e.g., control group); (2) studies that include different types of cancer (except studies that include both patients with breast cancer and patients with gynecological cancer); (3) studies that evaluate psychological interventions that include other types of dyads (e.g., mother-child dyad; patient-friend dyad); (4) case studies or qualitative studies; (5) studies undertaken in a palliative or end-stage context.

Search strategy

Studies were identified by searching multiple literature databases related to health and psychology throught EBSCOhost, including CINAHL, PsycInfo, Academic Search Complete, MEDLINE, PsycArticles, and Psychology and Behavioral Science Collection (from 1975 to January 2013). In order to avoid publication and source selection bias these database searchers were supplemented by additional hand-searching of recent literature reviews, unpublished articles and doctoral theses (through searching in Google and searching in abstracts of conferences and congresses). The key search terms were: (type of disease) breast cancer OR mastectomy; (intervention) couple therapy OR couple intervention OR psychological intervention OR couple counseling; (population) couples OR women and their partners OR women and their husbands. The search was not constrained by any geographic or linguistic factors.

As suggested by the Cochrane Collaboration the selection of studies for eligibility and data extraction was undertaken by two independent reviewers in order to reduce the 
likelihood of missed studies or errors in classification (Higgins \& Green, 2011). Any disagreements between reviewers were discussed and a consensus was reached.

\section{Results}

A total of 129 studies were identified from all databases and search methods: 56 from PsycInfo, 30 from Academic Search Complete, 15 from Psychology and Behavioral Sciences Collection, 15 from Medline, six from PsycArticles, six from CINAHL and one from hand-searching methods (see figure 1). Careful review of the retrieved titles identified 61 studies that were duplicates (i.e., they had the same title). The abstracts of the remaining 68 studies were screened and evaluated. From these, 55 studies were excluded (50 did not evaluate the efficacy of intervention, three were case studies and two did not include a comparison group) and 13 studies were selected for full-text review. The full-text review indicated that 11 met all criteria for inclusion; one of the 13 were excluded because it focused on another type of dyad (e.g., parent-child dyad) and one was excluded because it was a non-randomized study. Further review determined that two of the 11 remaining studies (Manne et al., 2005; 2007) had substantially overlapping samples from the same study, reducing the final sample of studies to ten.

The limited number of studies combined with the diversity and heterogeneity of the studies in terms of their characteristics, outcomes evaluated and instruments used raised significant concerns about the merits of performing a systematic meta-analysis (Alderson \& Green, 2002; Higgins \& Green, 2011). Instead, the focus of this paper is on summarizing the types of interventions, reviewing their components, describing their results, and providing recommendations for future systematic reviews.

[Figure 1 near here]

A summary of the characteristics of included studies is presented in Table 1 . Across studies, the total number of participating couples was $679(\operatorname{Min}=12$; $\operatorname{Max}=238)$, with 309 (Min = 8; Max = 120) participating in the experimental groups and $276(\mathrm{Min}=$ 3; $\operatorname{Max}=118$ ) in the comparison groups. The mean age for women was 50 (range: 40 to 54) and for men was 51 (range: 40 to 58). The mean length of the relationship was 22 years (range: 15 to 29). The target of the interventions in all included studies was couples in which the wife was diagnosed with non-metastatic breast or gynecological cancer (stage from 0 to IIIa). Participation rates were not reported in half of the studies, but the 
mean rate of participation for invited couples for studies that did report these data was $38 \%$ (range: $13 \%$ to $94 \%$ ).

[Table 1 near here]

All studies evaluated psychological interventions for couples coping with breast cancer. Two studies also included couples facing gynecological cancer since they have common characteristics. In breast and gynecological cancers women have similar treatments, and the cancers have a clear impact on their femininity and sexuality. For these reasons, similar psychosocial concerns arise for couples (Heinrichs et al., 2012; Scott et al., 2004).

All but one of the interventions in included studies were conducted with individual couples in couples' homes or at the hospital. The remaining study conducted the intervention in a group format (Manne et al., 2005; Manne, Ostroff, \& Winkel, 2007). Four studies compared couples intervention to treatment as usual or usual care (Baucom et al., 2009; Kayser, Feldman, Borstelmann, \& Daniels, 2010; Manne et al., 2005, 2007; Weisenthal, 2006), two to an active couple-based control group (Heinrichs et al., 2012; Zimmermann \& Heinrichs, 2011) and two to the delivery of psychoeducation and medical information about breast cancer and coping with cancer treatment (Naaman, 2008; Scott et al., 2004).

All interventions were delivered face-to-face; one included supplementary sessions that were delivered over the telephone (Scott et al., 2004). The number of sessions varied from four to 20 sessions, with a session length that varied from 60 to 120 minutes. All except one intervention was given on a weekly or a bi-weekly frequency. In one study, the sessions differed in frequency depending on what point in treatment the woman was (Scott et al., 2004).

In five studies, the interventions were delivered by psychologists or clinical psychologists (Baucom et al., 2009; Heinrichs et al., 2012; Naaman, 2008; Scott et al., 2004; Zimmermann \& Heinrichs, 2011), one were delivered by a psychologist and social worker (Weisenthal, 2006), two were delivered by therapists (Christensen, 1983; Manne et al., 2005, 2007), and one was delivered by a clinical social worker (Kayser et al., 2010). All studies followed a manualized intervention or a default protocol, and the majority included strategies to maintain treatment fidelity (Baucom et al., 2009; Christensen, 1983; Heinrichs et al., 2012; Kayser et al., 2010; Manne et al., 2005, 2007; Naaman, 2008; Scott et al., 2004). With respect to theoretical approach, the interventions were built on different 
conceptual foundations, such as cognitive-behavioral theory (Baucom et al., 2009; Heinrichs et al., 2012; Zimmermann \& Heinrichs, 2011), cognitive social processing theory (Manne et al., 2005, 2007), and attachment theory (Naaman, 2008). The remaining studies did not explicitly indicate the theoretical approach adopted.

The main outcomes assessed both for men and women were related to psychological, physical, marital and sexual functioning. For women, health indicators, such as illness-specific symptoms, were also assessed. Two studies did not report outcomes for partners (Kalaizti et al., 2007; Manne et al., 2005, 2007) and only one study (Naaman, 2008) examined physiological outcomes (namely, natural killer cell cytotoxicity). In terms of post-intervention assessment, two studies had only one followup (Christensen, 1983; Kalaitzi et al., 2007), four studies had two follow-ups (Baucom et al., 2009; Kayser et al., 2010; Manne et al., 2005, 2007; Naaman, 2008), and four had three follow-ups (Heinrichs et al., 2012; Scott et al., 2004; Weisenthal, 2006; Zimmermann \& Heinrichs, 2011). Follow-up timing ranged from post-intervention to 12 months.

The results obtained were mixed in terms of providing evidence for efficacy. Two studies found no significant differences between intervention and control groups on any of the dimensions evaluated (Kayser et al., 2010; Weisenthal, 2006). However, the remaining eight studies found significant differences between intervention and control groups for both women and their partners. More specifically, female participants in the intervention groups reported significantly better psychological well-being (Baucom et al., 2009; Christensen, 1983; Manne et al., 2005, 2007), more posttraumatic growth (Baucom et al., 2009; Heinrichs et al., 2012), better self-image (Baucom et al., 2009; Scott et al., 2004), better sexual functioning and higher sexual satisfaction (Christensen, 1983; Kalaitzi et al., 2007; Scott et al., 2004; Zimmermann \& Heinrichs, 2011), better coping and communication (Heinrichs et al., 2012; Scott et al., 2004) and fewer physical symptoms associated with breast cancer (Baucom et al., 2009).

For men, studies found significant benefits for the intervention groups on psychological well-being (Baucom et al., 2009), posttraumatic growth (Baucom et al., 2009; Heinrichs et al., 2012), coping and communication (Heinrichs et al., 2012) and sexual functioning (Christensen, 1983; Zimmermann \& Heinrichs, 2011). Both women and their partners in intervention groups reported improvements in relationship functioning (Baucom et al., 2009). 
Only one study compared the results of two treatment groups (Scott et al., 2004). The study found couple-based coping training was more effective than individual coping training in facilitating adaptation to cancer. Only two studies reported analyses of moderators of treatment (Kayser et al., 2010; Manne et al., 2005, 2007). Kayser et al. (2010) found that women with shorter length relationships and those receiving chemotherapy reported stronger improvements in their quality of life as a result of the couples-based intervention. Manne et al. $(2005,2007)$ found that women evaluating their partners as less supportive reported more physical impairment following the intervention than those with more supportive partners. Also women who reported using more emotional expression and emotional processing to cope with cancer also reported more benefits from the intervention. Unfortunately, only four studies reported the effect size of the results obtained for all of the dimensions evaluated (Baucom et al., 2009; Manne et al., 2005; Kayser et al., 2010; Scott et al., 2004; Zimmerman \& Heinrichs, 2011), and only one study reported the median interpolated effect size across all measures (Baucom et al., 2009).

\section{Discussion}

The main objective of the present systematic review was to analyze the content, the format, as well as the efficacy of psychological interventions for couples coping with breast cancer. Despite the existence of recent systematic reviews addressing the efficacy of psychological intervention for couples dealing with medical problems this is the first to evaluate couples interventions specifically for couples coping with breast cancer. In fact, contrary to previous systematic reviews and meta-analyses that include different types of cancer and chronic diseases, this systematic review is more focused and uses more rigorous inclusion and exclusion criteria. It includes only psychological interventions delivered for couples coping with breast cancer or gynecological cancer, in which the adult intimate partner is actively included (that is, the intervention has an explicit couple component). Inclusion criteria also required studies to have a comparison group. Adherence to the Cochrane Collaboration guidelines, the use of two independent researchers for reviewing eligibility and extracting data as well as efforts to diminish publication bias strengthen this systematic review. For these reasons, this review can inform the practice of psychological intervention for couples in the context of breast cancer. 
Overall, the results of this review suggest that psychological interventions for couples coping with breast cancer are effective, with eight of ten studies finding significant differences between the experimental and the control group in the expected direction. However, it is important to note that statistically reliable differences were not found in all of the outcomes. Also the significant differences found ranged from small to large effect sizes (ranging from $d=.02$ to 1.23 ). These widely varying results may reflect differences in the format and characteristics of interventions (e.g., number of sessions, the professional training of the individuals delivering the intervention) and the measures used to evaluate the dimensions studied. A wide range of therapeutic approaches was evident, and a number of studies did not specify the specific theoretical orientation underlying their interventions, which makes it difficult to evaluate the theoretical underpinnings of effective treatment. Future studies should present more information regarding this topic.

Interestingly, despite the diversity in many characteristics and the theoretical orientations underlying the interventions, the content of the psychological interventions is quite similar. Common elements across studies included: (1) a significant psychoeducational component designed to provide information about the cancer, the treatment and its side effects and to normalize psychological responses to cancer (Baucom et al., 2009; Christensen, 1983; Scott et al., 2004; Wiesenthal, 2006); (2) the promotion of emotional expression and social support (Baucom et al., 2009; Christensen, 1983; Kayser et al., 2010; Manne et al., 2005, 2007; Naaman, 2008, Scott et al., 2004; Wiesenthal, 2006); (3) interventions intended to promote sexual and body adaptation (Baucom et al., 2009; Christensen, 1983; Kalaitzi et al., 2007; Kayser et al., 2010; Manne et al., 2005, 2007; Scott et al., 2004; Wiesenthal, 2006; Zimmermann \& Heinrichs, 2011); and (4) the promotion of benefit finding, posttraumatic growth and meaning in life (Baucom et al., 2009; Wiesenthal, 2006). All the interventions included training in specific coping, problem-solving and communication skills despite the different theoretical approaches.

However, our review of studies indicates that similar content does not necessarily lead to similar results. This is not surprising since even when the intervention is manualized the therapists and their personal characteristics as well as the relationship between the therapists and the patients have an important role in the results obtained (Sprenkle, Davis, \& Lebow, 2009). Indeed, Norcross (2011) found that the therapy relationship influences the improvement of patients as much as the treatment adopted. 
Aspects of alliance in individual therapy, or more specifically, empathy by the therapist predicted treatment outcome consistently across different types of treatments (Elliott, Bohart, Watson, \& Greenberg, 2011). Also, Friedlander, Escudero, Heatherington, and Diamond (2011) studying the therapeutic alliance in couple and family therapy found that the development of a good alliance is fundamental for the success of psychotherapy, regardless of the type of treatment. Finally, another alliance-like quality in group interventions, group cohesion, has consistently been linked to therapy outcome (Burlingame, McClendon, \& Alonso, 2011).

Only two of the ten studies in this review provided data on the evaluation of the therapeutic alliance (Naaman, 2008; Scott et al., 2004). In both cases, therapeutic relationship factors were not explored as a potential source of influence on the efficacy of the intervention. The lack of attention to therapeutic alliance represents a gap in the study of efficacy of psychological interventions for couples coping with breast cancer. In fact, common factors that influence the outcomes of interventions, such as therapeutic alliance, therapist characteristics and group characteristics, are not being taken into account and their influence is not being explored.

There are other factors that may help explain the variation in results obtained across reviewed studies. First, study participants were very heterogeneous in terms of cancer characteristics. Studies included women with breast cancer with different stages, different histological typing, and different molecular diagnosis (e.g., estrogen receptor positive or negative). Also, important differences in cancer treatment have not been accounted for in previous studies. Given that surgical interventions and other treatments vary in their physical invasiveness and their characteristic side effects, it is important to control for the effect of these variables in studying treatment outcomes.

Second, many studies were characterized by small sample sizes (e.g., Baucom et al., 2009; Christensen, 1983; Naaman, 2008, Weisenthal, 2006), which limits statistical power and decreases the likelihood of seeing significant effects of the intervention group relative to the control or comparison group. Third, a number of studies did not have sufficient post-intervention follow-up evaluations to determine if results are maintained over time (Christensen, 1983; Kalaitzi et al., 2007; Kayser et al., 2010; Manne et al., 2005, 2007; Naaman, 2008). Fourth, in many studies the experimental group was compared to a treatment-as-usual group or standard services (Baucom et al., 2009; Kayser et al., 2010; Manne et al., 2005, 2007; Wiesenthal, 2006). The lack of an experimental control group may obscure intervention effects because, as Kayser et al. (2010) 
documents, some patients not in the intervention group may have access to psychological interventions outside the study. For these reasons, the recruitment of more homogenous and larger samples, the inclusion of more follow-up evaluations and the use of an experimental control group are important aspects that should be taken into consideration in future research.

Finally, the majority of studies failed to include variables that might moderate intervention outcomes. Only two studies evaluated the role of potential moderators. Manne et al. $(2005,2007)$ evaluated the moderating role of partners' support, physical impairment, emotional expression and emotional processing. Kayser et al. (2010) evaluated the moderating role of relationship length and medical treatment. In future research, it will be important to consider other types of moderators, such as demographic or clinical characteristics (e.g., stage of disease, type of surgery, type of cancer treatment) and psychological variables (e.g., perceived social support, marital satisfaction). Some of the latter variables, which are focused on couple interaction and couple-well-being, may also be useful to examine as potential mediators of therapeutic outcomes. Although some of these variables (e.g., stage of disease or type of surgery) were reported in many studies they were not included in the analyses presented.

Another important factor to consider in future research is the possible impact of initial functioning on treatment response. Only one study (Manne et al., 2005) controlled for the effects of pre-intervention scores on treatment results. The inclusion of potential moderators in analyses is essential in order to identify for whom the intervention is more effective.

It is important to note that the patients in the studies varied with respect to the phase of the disease in which the intervention was delivered (diagnosis, treatment or survival). In fact, some studies had very broad inclusion criteria that resulted in patients in many different phases of the treatment process. Some studies specified an inclusion criterion based on months of diagnosis or time from surgery, ranging from two to 12 months (Kayser et al., 2010; Christensen, 1983; Naaman, 2008). Other studies used active treatment (Kayser et al., 2010; Scott et al., 2004) or recently finished treatment as an inclusion criterion (Kalaitzi et al., 2007; Naaman, 2008). In future research, it will be important to control or explicitly consider phase of the disease in order to understand when psychological interventions are most effective. Reducing variability by having narrower inclusion criteria regarding phase of treatment (e.g., recently diagnosed breast cancer or after having undergone surgery) might improve future studies by reducing 
within-group variance that hinders statistical power and by reducing the existence of potential confounding factors (Baucom et al., 2009; Heinrichs et al., 2012; Manne et al., 2005, 2007; Zimmerman \& Heinrichs, 2011; Wiesenthal, 2006). Designing studies with narrower inclusion criteria is also likely to increase the existence of shared experiences and commonalities among participants, which might have important intervention effects, such as yielding greater cohesion and satisfaction or allowing the group to work faster (Perrone \& Sedlacek, 2000).

More sophisticated data analytic approaches should be integrated in future research, particularly to explore dyadic influences. The conventional analyses that have been used to study couples are not adequate to fully understand the complex, dyadic influences of couples' relationship, since these analyses assume the independence of observations in the dependent variable (e.g., ANOVA). Recognizing that data from couples is nonindependent (i.e., it is linked and correlated), it is essential to use data analytic strategies such as the Actor-Partner Independence Model (APIM) that captures direct and indirect effects between partners and accounts for complicated dependencies in the data (Cook \& Kenny 2005; Kenny, Kashy, \& Cook, 2006). The APIM is a useful framework for the kind of analyses in which it is important to treat the dyad rather than the individual as the unit of analysis. Importantly, it can be expanded to allow for the evaluation of inter-partner influence across time, which may be an important factor in psychological interventions for couples (Cook \& Snyder, 2005). At a minimum, it is important to evaluate and report the non-patient partners' experiences and functioning. This is something that the majority of studies failed to do and that future research should take into account.

Despite the limitations of the studies presented here, they are important because they provide information about the power of psychological interventions for couples coping with breast cancer. This review provides information and recommendations that can guide future research by highlighting important research directions and past limitations. The studies that did not show statistically significant interventions effects when considered in isolation are important because they suggest some potential benefits and trends, in the expected direction, with non-ignorable effect sizes. As more studies accumulate, these effect sizes can be aggregated into meta-analytic studies that take advantage of greater statistical power. On the whole, the studies reviewed suggest that psychological intervention for couples coping with breast cancer can be beneficial for some specific couples. Future research should address for whom and under what 
circumstances couple interventions are more effective, and begin to address the critical process questions of why and how they are effective.

The results presented in this review are in line with previous systematic reviews and meta-analysis that show that psychological interventions for couples coping with cancer and other chronic diseases produce benefits for both patients and their partners (Badr \& Krebs, 2012; Baik \& Adams, 2011; Hopkinson, Brown, Okamoto, \& AddingtonHall, 2012).

\section{Limitations}

It is important to keep in mind two limitations when considering the results of this review. First, the sample of studies is small, since it includes only ten studies. The small number of studies attests to the fact that the efficacy of psychological interventions in this sphere is understudied. Couple-based interventions, in particular, are a relatively new approach among psychosocial interventions for breast cancer. For these reasons, the results must be interpreted with caution and further studies are needed.

Second, as in all systematic reviews, there is the risk of reporting bias, which means that of the primary focus has been on studies published in identifiable sources; there is always the risk of not accessing relevant studies that are unpublished or were not presented in traditional outlets. Such studies may be more likely to not have significant results that indicate efficacy for the intervention. Non-significant results are as important as significant results and should also be disseminated. In order to reduce selection biases we carefully searched for and included unpublished studies. We also made no constraints regarding geographic and linguistic criteria.

\section{Conclusions and implications for practice}

In this review, we discussed the utility and the efficacy of psychological interventions for couples coping with breast cancer. Although there are previous systematic reviews regarding couple intervention in the oncological context, this is the first to focus only on couples coping specifically with breast cancer. The limited number of studies conducted in this area suggests that psychological interventions for this population are effective with benefits for both women and their partners. These benefits are evident not only in psychological variables (e.g., anxiety, depression and emotion regulation) but also in relational variables (e.g., social support, couples' communication and sexual functioning). 
However, more efforts are needed to identify and to understand the differential responses to treatment and the processes and mechanisms of change involved in these interventions. Such efforts would help identify for whom and under what circumstances particular interventions are more effective, provide important information about why they might be effective, and clarify which components are necessary to achieve efficacy.

As mentioned before, breast cancer has an impact not only on affected women but also on their partners. However, this impact and the needs of caregivers are not always recognized and identified by care recipients and health professionals. For this reason the assessment of caregivers' well-being should become more routine. More generally, partners should be incorporated into psychosocial cancer care in order to identify their needs and to help preserve their well-being and to facilitate their vital roles as providers of support for the patients. Psychoeducation about cancer, its treatments and it psychosocial impact seems essential to both parties to assist in relieving individual and couple stress.

This systematic review contributes in raising awareness to the value of including partners of women with breast cancer in psychological interventions. It also emphasizes the need of incorporate couple-based interventions with proven efficacy into the standard care of women with breast cancer.

\section{References}

Alderson, P., \& Green, S. (2002). Cochrane Collaboration open learning material for reviewers [version 1.1]. Retrieved from: http://www.cochranenet.org/openlearning/PDF/Openlearning-full.pdf.

Anagnostopoulos, F., Slater, J., \& Fitzsimmons, D. (2010). Intrusive thoughts and psychological adjustment to breast cancer: Exploring the moderating and mediating role of global meaning and emotional expressivity. Journal of Clinical Psychology in Medical Settings, 17, 137-149. doi: 10.1007/s10880-010-9191-6

Badger, T. A., Segrin, C., Dorros, S. M., Meek, P., \& Lopez, A. M. (2007). Depression and anxiety in women with breast cancer and their partners. Nursing Research, 56, 4453.

Badr, H., \& Krebs, P. (2012). A systematic review and meta-analysis of psychosocial interventions for couples coping with cancer. Psycho-oncology. doi: 10.1002/pon.3200. 
Baider, L., Ever-Hadani, P., Goldzweig, G., Wygoda, M. R., \& Peretz, T. (2003). Is perceived family support a relevant variable in psychological distress? A sample of prostate and breast cancer couples. Journal of Psychosomatic Research, 55, 453-460.

Baik, O. M., \& Adams, K. B. (2011). Improving the well-being of couples facing cancer: A review of couple-based psychosocial interventions. Journal of Marital \& Family Therapy, 37, 250-266. doi: 10.1111/j.1752-0606.2010.00217.x

Baucom, D. H., Kirby, J. S., Pukay-Martin, N. D. Porter, L. S., Fredman, S. J., Gremore, T. M., ... Atkins, D. (2012). Men's psychological functioning in the context of women's breast cancer. Journal of Marital and Family Therapy, 38, 317-329. doi: 10.1111/j.17520606.2009.00133.x

Baucom, D., Porter, L., Kirby, J., Gremore, T., Wiesenthal, N., Aldridge, W., ... Keefe, F. (2009). A couple-based intervention for female breast cancer. Psycho-Oncology, 18, 276-283. doi: 10.1002/pon.1395

Burlingame, G. M., McClendon, D. T., \& Alonso, J. (2011). Cohesion in group therapy. In J. C. Norcross (Ed.), Psychotherapy relationships that work: Evidence-based responsiveness (pp. 110-131). Oxford: Oxford University Press.

Caress, A. L., Chalmers,K., \& Luker, K. (2009). A narrative review of interventions to support family carers who provide physical care to family members with cancer. International Journal of Nursing Studies, 46, 1516-1527.

Christensen, D. N. (1983). Post mastectomy couple counseling: An outcome study of a structured treatment protocol. Journal of Sex and Marital Therapy, 9, 266-275.

Cook, W. L., \& Kenny, D. A. (2005). The actor-partner independence model: A model of bidirectional effects in developmental studies. International Journal of Behavioral Development, 29, 101-109.

Cook, W. L., \& Snyder, D. K. (2005).Analyzing nonindependent outcomes in couple therapy using the actor partner interdependence model. Journal of Family Psychology, $19,13-141$.

Elliot, R., Bohart, A. C., Watson, J. C. \& Greenberg, L. S. (2011). Empathy. In J. C. Norcross (Ed.), Psychotherapy relationships that work: Evidence-based responsiveness (pp. 132-152). Oxford: Oxford University Press.

Feeney, B., \& Collins, N. (2003). Motivations for caregiving in adult intimate relationships: Influences on caregiving behavior and relationship functioning. Personality and Social Psychology Bulletin, 29, 950-968. 
Ferlay, J., Steliarova-Foucher, E., Lortet-Tieulent, J., Rosso, S., Coebergh, J., Comber, H., ... Bray, F. (2013). Cancer incidence and mortality patterns in Europe: Estimates for 40 countries in 2012. European Journal of Cancer. doi:10.1016/j.ejca.2012.12.027

Friedlander, M. L., Escudero, V., Heatherington, L., \& Diamond, G. M. (2011). Alliance in couple and family therapy. In J. C. Norcross (Ed.), Psychotherapy relationships that work: Evidence-based responsiveness (pp.92-109). Oxford: Oxford University Press.

Heinrichs, N., Zimmerman, T., Huber, B., Herschbach, P., Russell, D., \& Baucom, D. (2012). Cancer distress reduction with a couple-based skills training: A randomized controlled trial. Annals of Behavioral Medicine, 43, 239-252. doi: 10.1007/s12160-0119314-9

Higgins, J. P., \& Green, S. (2011). Cochrane Handbook for Systematic Reviews of Interventions [version 5.1.0]. Retrieved from: www.cochrane-handbook.org.

Hopkinson, J. B., Brown, J. C., Okamoto, I., \& Addington-Hall, J. M. (2012). The effectiveness of patient-family carer (couple) intervention for the management of symptoms and other health-related problems in people affected by cancer: A systematic literature search and narrative review. Journal of Pain and Symptom Management, 43, 111-142. doi: 10.1016/j

Kalaitzi, C., Papadopoulos, V. P., Michas, K., Vlasis, K., Skandalakis, P., \& Filippou, D. (2007). Combined brief psychosexual intervention after mastectomy: Effects on sexuality, body image, and psychological well-Being. Journal of Surgical Oncology, 96, 235-240.

Kayser, K., Feldman, B. N., Borstelmann, N. A., \& Daniels, A. A. (2010). Effects of a randomized couple-based interventionon quality of life of breast cancer patients and their partners. Social Work Research, 34, 20-32. doi: 10.1093/swr/34.1.20

Kenny, D. A., Kashy, D. A., \& Cook, W. L. (2006). Dyadic data analysis. New York: The Guildford Press.

Kim, Y., Carver, C. S., Deci, E. L., \& Kasser, T. (2008). Adult attachment and psychological well-being in cancer caregivers: The meditational role of spouses' motives for caregiving. Health Psychology, 27, 144-154. doi: 10.1037/02786133.27.2(Suppl.).S144

Lethborg, C. E., Kissane, D., \& Burns, W. I. (2003). 'It's not the easy part': The experience of significant others of women with early stage breast cancer, at treatment completion. Social Work in Health Care, 37, 63-85. 
Manne, S. L., Ostroff, J. S., Winkel, G., Fox, K., Grana, G., Miller, E., ... Fazier, T. (2005). Couple-focused group intervention for women with early stage breast cancer. Journal of Consulting \& Clinical Psychology, 73, 634-646.

Manne, S., Ostroff, J., Norton, T., Fox, K., Goldstein, L., \& Grana, G. (2006). Cancerrelated relationship communication in couples coping with early stage breast cancer. Psycho-Oncology, 13, 234-247.

Manne, S., Ostroff, J. S., \& Winkel, G. (2007). Social-cognitive processes as moderators of a couple-focused group intervention for women with early stage breast cancer. Health Psychology, 26, 735-744.

Martire, L. M., Schulz, R., Helgeson, V. S., Small, B. J., \& Saghafi, E. M. (2010). Review and meta-analysis of couple-oriented interventions for chronic illness. Annual Behavior Medicine, 40, 325-342. doi: 10.1007/s12160-010-9216-2

Montazeri, A., Vahdaninia, M., Harirchi, I., Ebrahimi, M., Khaleghi, F., \& Jarvandi, S. (2008). Quality of life in patients with breast cancer before and after diagnosis: An eighteen months follow-up study. BioMed Central Cancer, 8, 330-336. doi: $10.1186 / 1471-2407-8-330$

Moreira, H., \& Canavarro, M. C. (2010). A longitudinal study about the body image and psychosocial adjustment of breast cancer patients during the course of the disease. European Journal of Oncology Nursing, 14, 263-270. doi:10.1016/j.ejon.2010.04.001

Moreira, H., \& Canavarro, M., C. (2013). Psychosocial adjustment and marital intimacy among partners of patients with breast cancer: A comparison study with partners of healthy women. Journal of Psychosocial Oncology, 31, 282-304.

Naaman, S. (2008). Evaluation of the clinical efficacy of emotionally focused couples therapy on psychological adjustment and natural killer cell cytotoxicity in early breast cancer [Unpublished doctoral dissertation]. University of Ottawa, Canada.

Norcross, J. C. (Ed.). (2011). Psychotherapy relationships that work: Evidence-based responsiveness. Oxford: Oxford University Press.

Northouse, L. L., Katapodi, M., Song, L., Zhang, L., \& Mood, D. W. (2010). Interventions with family caregivers of cancer patients: Meta-analysis of randomized trials. Cancer Journal Clinic, 60, 317-339. doi: 10.3322/caac.20081

Perrone, K. M., \& Sedlacek, W.E. (2000). A comparison of group cohesiveness and client satisfaction in homogenous and heterogenous groups. The Journal for Specialists in Group Work, 25, 243-251 
Regan, T. W., Lambert, S. D., Girgis, A., Kelly, B., Kayser, K., \& Turner, J. (2012). Do couple-based interventions make a difference for couples affected by cancer: A systematic review. BioMed Central Cancer. doi:10.1186/1471-2407-12-279.

Sbitti, Y., Kadiri, H., Essaidi, I., Fadoukhair, Z., Kharmoun, S., ... Errihani, H. (2011). Breast cancer treatment and sexual dysfunction: Moroccan women's perception. BioMed Central Women's Health, 11(29), 1-5.

Scott, J. L., Halford, W. K., \& Ward, B. G. (2004). United we stand? The effects of a couple-coping intervention on adjustment to early stage breast or gynecological cancer. Journal of Consulting and Clinical Psychology, 72, 1122-1135.

Silva, S., Bettencourt, D., Moreira, H., \& Canavarro, M. C. (2011). Qualidade de vida de mulheres com cancro da mama nas diversas fases da doença: o papel de variáveis sociodemográficas, clínicas e das estratégias de coping enquanto factores de risco/protecção [Quality of life of women with breast cancer at different phases of the disease: the role of sociodemographic, clinical variables and coping strategies as factors of risk/protection]. Revista de Saúde Pública, 29, 64-76.

Sprenkle, D. H., Davis, S. D., \& Lebow, J. L. (2009). Common factors in couple and family therapy: The overlooked foundation for effective practice. New York: The Guilford Press.

Stiell, K., Naaman, S., \& Lee, A. (2007). Couples and chronic illness: An attachment perspective and emotionally focused therapy interventions. Journal of Systemic Therapies, 26, 59-74. doi: 10.1521/jsyt.2007.26.4.59

Taylor, S., Harley, C., Ziegler, L., Brown, J., \& Velikova, G. (2011). Interventions for sexual problems following treatment for breast cancer: a systematic review. Breast Cancer Research and Treatment, 130, 711-724. doi: 10.1007/s10549-011-1722-9

Ussher, J. M., Perz, J., Hawkins, Y., \& Brack, M. (2009). Evaluating the efficacy of psycho-social interventions for informal carers of cancer patients: A systematic review of the research literature. Health Psychology Review, 3, 85-107. doi: $10.1080 / 17437190903033401$

Ussher, J. M., Perz, J., \& Gilbert, E. (2012). Information needs associated with changes to sexual well-being after breast cancer. Journal of Advanced Nursing, 69, 327-337. doi: $10.1111 / \mathrm{j} .1365-2648.2012 .06010$

Wagner, C. D., Bigatti, S. M., \& Storniolo, I. N. (2006). Quality of life of husbands of women with breast cancer. Psycho-Oncology, 15, 109-120. 
Weihs, K. I., Enright, T. M., \& Simmens, S. J. (2008). Close relationships and emotional processing predict decreased mortality in women with breast cancer: Preliminary evidence. Psychosomatic Medicine, 70, 117-124.

Wiesenthal, N. L. (2006). Posttraumatic growth in a cognitive behavioral couple intervention for women with early stage breast cancer. [Unpublished doctoral dissertation]. University of North Carolina, Chapel Hill.

Zimmermann, T., \& Heinrichs, N. (2011). Auswirkungen einer psychoonkologischen intervention für paare auf die sexualität bei einer brustkrebserkrankung der frau. [Effects of a psychosocial intervention for couples on sexuality when the woman has breast cancer]. Zeitschrift für Gesundheitspsychologie, 19, 23-34. doi: 10.1026/09438149/a000037 